\title{
MODELLING OF MATERIAL PARAMETER UNCERTAINTY OF RESONANCE WOOD USED IN VIOLINS USING THE INTERVAL FIELD
}

\author{
Maurice Imholz ${ }^{1}$, Dirk Vandepitte ${ }^{1}$ and David Moens ${ }^{1}$ \\ ${ }^{1}$ KU Leuven, Department of Mechanical Engineering \\ Celestijnenlaan 300, B-3001, Leuven \\ e-mail: maurice.imholz@kuleuven.be - dirk.vandepitte@kuleuven.be - david.moens@kuleuven.be
}

Keywords: uncertainty analysis, interval methods, interval fields

\begin{abstract}
The interval field method can be used to model spatial uncertainty in finite element models. When analysing spatial uncertainty, dependency is an important quantity to consider, as it is physically implausible to assume a quantity is independent in locations that are close to each other. The interval field method was introduced to join this concept of dependency with classical interval analysis, omitting the need to define probability distributions. This paper will apply the theory of interval field (IF) to the dynamical properties of a violin top plate, with uncertain stiffness and density.
\end{abstract}




\section{INTRODUCTION}

The construction of musical instruments is an interesting field for numerical modelling. Not only because numerical modelling is still almost non-existent in this field, but also because due to the sensitivity of the human eye, almost all aspects than can be modelled accurately from the raw material up to the response of the final product are relevant. On the other hand, many effort has already been taken in defining numerical models for all kinds of instruments, and there is still much to do, because accurately modelling sound generated by instruments is notoriously difficult.

Some instruments can be mass produced, of which guitars are the best example, but in most instruments, a lot of handwork is still needed to obtain the desired end result. The raw material used in the construction is of vital importance to the sound, and much effort goes into selecting the best material. However, this too is mostly done using only the naked eye to assess material quality. This leaves a large amount of uncertainty on the material's structural properties, which in turn affects the structural output of the material, and ultimately the sound generated.

This paper considers the violin as instrument of interest, and attempts to quantify the uncertainty on relevant natural frequencies, originating from the uncertainty on the wood stiffness and density. Both parameters are assumed to vary over the domain of a violin top plate, leading to a field-based approach of the uncertain parameters. The reader should consider this work as part of a full sound model of a violin, in which much research has been conducted already. Important for subsequent analysis is that the uncertainty of the frequencies is captured as accurately as possible. If not, possible conservatism in the results could multiply, leading to useless results.

\section{VIOLIN BODY CONSTRUCTION}

The violin body accounts for the primary source of sound. The wooden corpus consists of two panels, the violins back and top panel. The panels are connected by the ribs of the instrument, enclosing a body of air. The f-shaped holes in the top plate allow air to flow in and out of the body, producing the core sound of the violin.

The top plate is made from quarter cut spruce (picea abies), sawn as a single piece, and is about $3 \mathrm{~mm}$ thick at the thickest point. The quarter-cut sawing technique produces planks with the growth rings almost perpendicular to the plank plane. This produces strong and light planks, but is more expensive to make as relatively few quarter-cut planks can be made from a single tree. The wood grains lie along the violins longitudinal axis.

The back plate is generally made from figured maple, and can be both a single piece or a two-pieced part. Two-pieced plates are made by sawing a single wedge from a tree into two slender wedges and gluing them together at the wide side. Because they originate from the same wedge, the two halves are visually almost identical, producing a symmetrical back plate. Single-pieced back plates can be both quarter-cut or slab-cut. Slab cut wood originates from sawing a tree all the way through. Because the alignment of the material axis vary over the plank, slab-cut backs are more sensitive and tend to bend more easily during the violins life.

The ribs are made from $1 \mathrm{~mm}$ thick maple wood, bended to follow the violins characteristic shape.

Within the violin body, a slender bar of spruce called the bass bar is attached to the top plate adding stiffness in the longitudinal direction. The bass bar is always attached with a slight offset from the middle, removing the symmetrical behaviour of the top plate.

Lastly, a small piece of spruce, the sound post, is put between the f-holes that connects the 

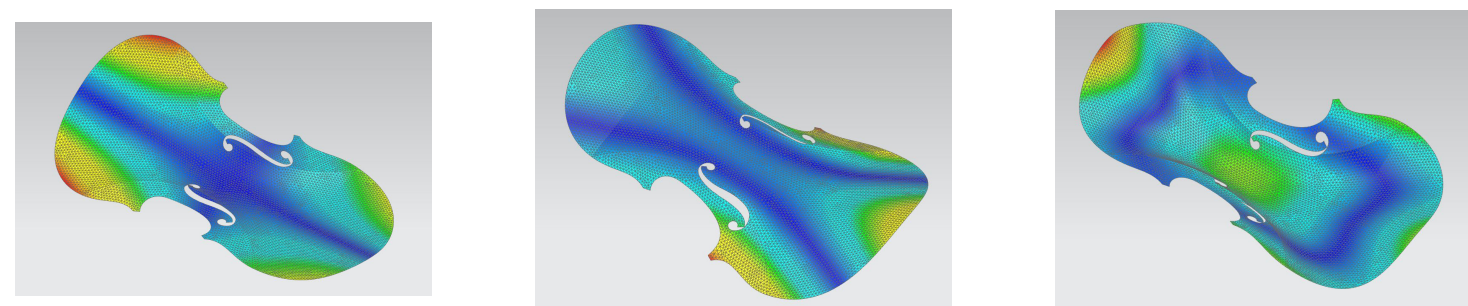

Figure 1: Tuning modes of the violin top plate

top and back plate, usually not glued in place, but clamped in between the plates, accounting for extra support of the top plate. Also placed eccentrically with respect to the top plates line of symmetry, the symmetry of the violin is further distorted.

For the purpose of this paper, only the top plate will be considered. Research has proven that for the characteristic modes of the violin body, the top plate is usually exhibiting the largest deformation.

\section{MODAL ANALYSIS}

The model is used in a modal analysis to characterise the structural behaviour of the violin top plate. The chain between violin body characteristics and sound production is notoriously hard to model accurately and has been subject of much research already, including work on bridge and snare mobility ([1, 2]), analysis of damping factors ([4, 5]) and modal characterisation of complete string instruments $([7,6])$ Earlier work on characterising the mechanics of the violin body already showed that not some structural modes are more effective at radiating sound than others ([3]). The effectiveness of a mode can be characterized in two ways:

- Modal displacement at the bridge: the string vibration is passed to the body through the bridge, placed in between the f-holes. If the mode shape exhibits a nodal line at the bridge, it is difficult to directly excite through exciting the strings, reducing sound radiation capability.

- Degree of asymmetry of the mode shape: with every mode shape a volume difference of the captured body of air is associated. Due to their symmetry, some heavily vibrating modes only lead to local motion of the air within the cavity, without actual air motion through the f-holes. Typically, highly symmetrical modes show this behaviour. More asymmetrical modes with more relative volume difference are therefore more effective at radiating sound.

Based on this fact, only a small set of modes is truly interesting to look at. During the construction process, most luthiers perform a plate tuning of both the top and back plate, specifically targeting one mode and tuning it to a desired value ([8]). Most luthiers use a manual process for this, holding the plate between thumb and index finger at a node and then gently tapping the plate at an antinode, producing an audible frequency associated to a single mode. By locally carving away some wood, the right frequency can be obtained. The modes mostly used to tune the top plate are the first, second and fifth structural mode, images of which are includes in figure 1 .

The first mode correspons to the first torsional mode of a flat plate, and is situated at about 60 $\mathrm{Hz}$. The second mode corresponds to the first bending mode. This mode is highly influenced by the anisotropic character of the wood, which can be seen from the curved nodal lines running 
across the plate. This mode's frequency is about $100 \mathrm{~Hz}$. The fifth mode is a bending mode with synchronic bending in $\mathrm{x}$ - and $\mathrm{y}$-direction, creating a trampoline-like movement. It is situated around $220 \mathrm{~Hz}$. This mode is closely related to the A0-mode of the full violin body. This important mode of the full violin exhibits opposite movement of top and back plate, leading to a large volume difference of the enclosed air, leading to large air movement through the f-holes. This mode is often referred to as the 'breathing'-mode. This mode is given in figure 2.

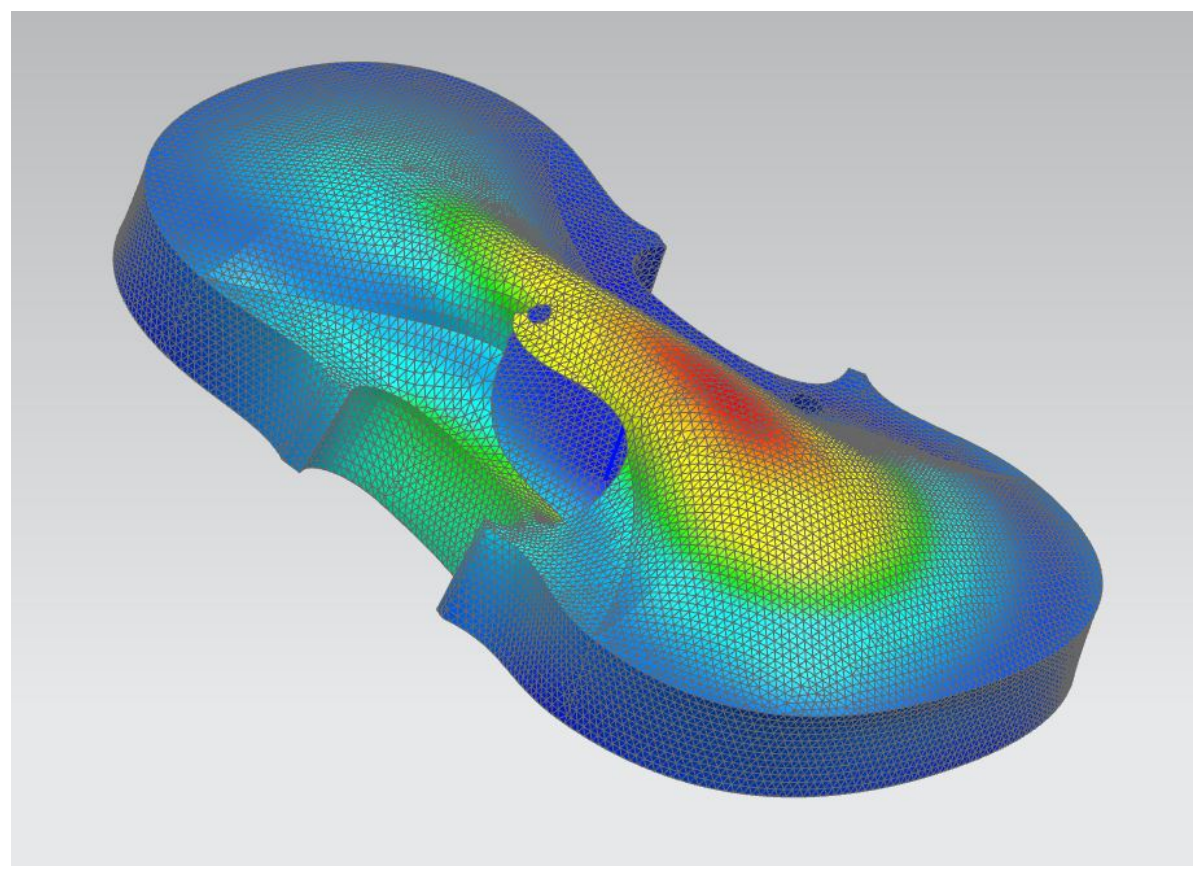

Figure 2: breathing mode of the violin body.

The model of the violin plate measures $210 \mathrm{~mm}$ at its widest and $356 \mathrm{~mm}$ at its longest point and consists of 15000 shell elements. The thickness is taken as $2.5 \mathrm{~mm}$ throughout.

With respect to possible automatisation of violin construction, the manual process of plate tuning is obviously the hardest to do. This analysis therefore assumes a constant thickness but allows a certain variation of the structural properties of the wood, as these are hard to determine for a violin maker. The output will be the frequency variation of the three tuning modes described above. However, not only the variation of the modes indiviually is important to observe, but also the shape of the combined uncertainty region in the $\left(f_{1}, f_{2}, f_{5}\right)$-domain, as the sound produced by the violin results of an interaction of these and probably even more modes. It is therefore very important to, as a start, accurately estimate an uncertain region of these mode frequencies, which can then be used in subsequent analysis of a full violin body analysis.

\section{WOOD VARIABILITY}

The primary use of wood in engineering purposes is in construction, for which only the structural properties are of interest. For use in musical instruments, the acoustic properties are the main source of interest. The term resonance wood is the term for all wood that is used for acoustical purposes, i.e. in musical instruments. The characterisation of resonance wood in this chapter mainly focusses on its orthotropic nature, and the different sources of variability. These 
sources will then be translated to a physically inspired representation of material uncertainty in the wood.

Wood is considered a nominally orthotropic material with cylindrical orthotropy. It is characterized by a density $\rho$, and three Moduli of Elasticity $\mathbf{E}_{\mathbf{L}}, \mathbf{E}_{\mathbf{R}}$ and $\mathbf{E}_{\mathbf{T}}$, representing the stiffness in the longitudinal, radial and tangential direction. Assuming quarter cut plates, the tangential direction is located perpendicular to the plate and is therefore of no interest in this analysis. Derived quantities are the speed of sound in longitudinal and radial direction, given by eq. 1 and 2. Specifically for resonance wood, $\mathbf{V}_{\mathbf{L L}}$ is quite high with respect to construction wood, indicating a large desired value for $\mathbf{E}_{\mathbf{L}}$ combined with a low density ([9]). On the radial stiffness, less conclusive results are known, but a relatively low value is generally preferred.

$$
\begin{gathered}
V_{L L}=\sqrt{\frac{E_{L}}{\rho}} \\
V_{R R}=\sqrt{\frac{E_{R}}{\rho}}
\end{gathered}
$$

If we look closely to the microstructure of wood, we observe tubular cells with a very large length-to-width ratio. In the case of spruce this is even larger than 100 [10]. The cell walls make up for almost all of the cells stiffness and are essentially a fibre-reinforced matrix. These fibres form a helix-shape around the cell with a varying angle called the microfibril angle (MFA). If the angle approaches zero, the fibres align with the cells main axis, maximizing the longitudinal stiffness and minimizing the radial stiffness, whereas if the angle increases, the stiffness of the matrix becomes increasingly dominant, reducing the longitudinal stiffness but increasing the radial stiffness. As a result, resonance wood is characterized by a small average MFA of 10 or less. Moreover, research has showed that a small difference between the average MFA of latewood compared to earlywood is beneficial for the acoustic properties, as this leads to a more homogeneous sound velocity $([11,12])$. A certain degree of continuity of this quantity over the violin plate is therefore desired.

\section{WOOD SELECTION}

Mostly, the selection process is based on visual techniques combined with experience to identify the optimal wood to be used in their instruments $([10,13,14])$. Their interest specifically goes out towards the annual ring pattern and wood colour. Narrow rings with good consistency over the length of the grains is desired, also because of aesthetic reasons. Also, a bright colour indicates lighter wood which is preferred as well. Overall, one can say that the violin makers selection process favors a low density and an impurity-free wood specimen over acoustical properties, which are much more difficult to assess by using only the senses. It has been shown that $\mathbf{E}_{\mathbf{R}}$ is proportional to the percentage of latewood present in the plate ([14]). Latewood is defined as the wood the tree grows in summer/autumn. Improving the trees strength, the density is more than twice the density of so called earlywood. Resonance wood usually has a latewood percentage of under $20 \%$, suggesting the preference of a low $\mathbf{E}_{\mathbf{R}}$.

\section{SPATIAL VARIABILITY}

Many literature exists with data on the variability of structural parameters of spruce ([18, 17, 16]), but most of these are defined on macro scale and derived from measuring entire wood 
planks. Focus on meso-level (i.e. variability between grains or along a single grain) is less abundant. We know wood consists of slender-shaped cells with very stiff cell walls, composed of a matrix-reinforced composite. The MFA is key in this definition as this determines the longitudinal and transverse stiffness of the cells and therefore of the whole material. Good correlation has been observed between the continuity of this parameter over the material and the acoustical quality of the resonance wood ([15]). Moreover, a large degree of anisotropy has the same positive effect on the quality, suggesting a small overall value for the MFA.

Research indicates that the stiffness is heavily dependent of the MFA, showing a linear decrease of longitudinal stiffness and an increase of radial stiffness with increasing MFA. Based on this reasoning, we assume the following dependency of the stiffnesses with respect to density and MFA, in each point on the top plate (eq. 3):

$$
\begin{aligned}
& E_{L}=E_{L, 0} \cdot\left(1+C_{\rho}\left(\rho-\rho_{0}\right)\right) \cdot\left(1-C_{\theta}\left(M F A-M F A_{0}\right)\right) \\
& E_{R}=E_{R, 0} \cdot\left(1+C_{\rho}\left(\rho-\rho_{0}\right)\right) \cdot\left(1+C_{\theta}\left(M F A-M F A_{0}\right)\right)
\end{aligned}
$$

We will assume the density and the MFA are not correlated, an assumption validated by literature ([19]), in which strong correlation between these quantities could not be proven. Note that for positive $C_{\theta}, E_{L}$ decreases as MFA increases, whereas the radial stiffness $E_{R}$ increases. For the purpose of this numerical exercise, we assume the density and the microfibrillar angle have similar effect on the final value of the stiffness (literature shows values between $1 / 3$ and $2 / 3$ ). Based on the ranges of $\rho$ and MFA, the values of the constants for spruce in equation 3 are given below in equation 4

$$
\begin{aligned}
& E_{L}=12 \cdot 10^{9} \frac{\mathrm{N}}{\mathrm{m}^{2}} \cdot\left(1+2.78 \cdot 10^{6} \frac{\mathrm{mm}^{3}}{\mathrm{~kg}}\left(\rho-\rho_{0}\right)\right) \cdot\left(1-0.0417\left(M F A-M F A_{0}\right)\right) \\
& E_{L}=0.9 \cdot 10^{9} \frac{\mathrm{N}}{\mathrm{m}^{2}} \cdot\left(1+2.78 \cdot 10^{6} \frac{\mathrm{mm}^{3}}{\mathrm{~kg}}\left(\rho-\rho_{0}\right)\right) \cdot\left(1-0.0417\left(M F A-M F A_{0}\right)\right)
\end{aligned}
$$

with MFA expressed in degrees. The density is the quantity on which the selection process of luthiers is mostly focused. As previously stated, light wood with a low contribution of latewood is preferred. The percentage of latewood can be relatively accurately estimated by the trained eye, so we assume no more than $20 \%$ intra-variability on the density in all directions. However, the density of the wood is expected to be more constant in the longitudinal direction than in the radial direction, as changes in weather conditions manifest themselves in the radial direction. We will therefore account for a higher dependency in the longitudinal direction than in the radial direction. The MFA is obviously much more difficult to notice with the blind eye. Fact is that Norway spruce (Picea abiens) has a very small average MFA with respect to other wood species, to which it largely owes its preferred use in violin building. The MFA is known to decrease with the age of the growth ring, and also with tree height [11]. The decrease rate is however subject to uncertainty. To express the MFA in each point, the following equation is used (equation 5):

$$
M F A^{I}(x, y)=\left(\theta_{2}^{I}-\theta_{1}^{I}\right) \cdot\left(\frac{x}{210 m m}\right)^{p_{1}^{I}}+\Delta \theta^{I} \cdot\left(\frac{y}{356 m m}\right)^{p_{2}^{I}}+\theta_{1}^{I}
$$

In this equation, $\theta_{1}$ and $\theta_{2}$ are the MFA at the lower corners of the plank from which the plate is made, $\Delta \theta$ is the change in MPA across the longitudinal axis of the plate. To all of 


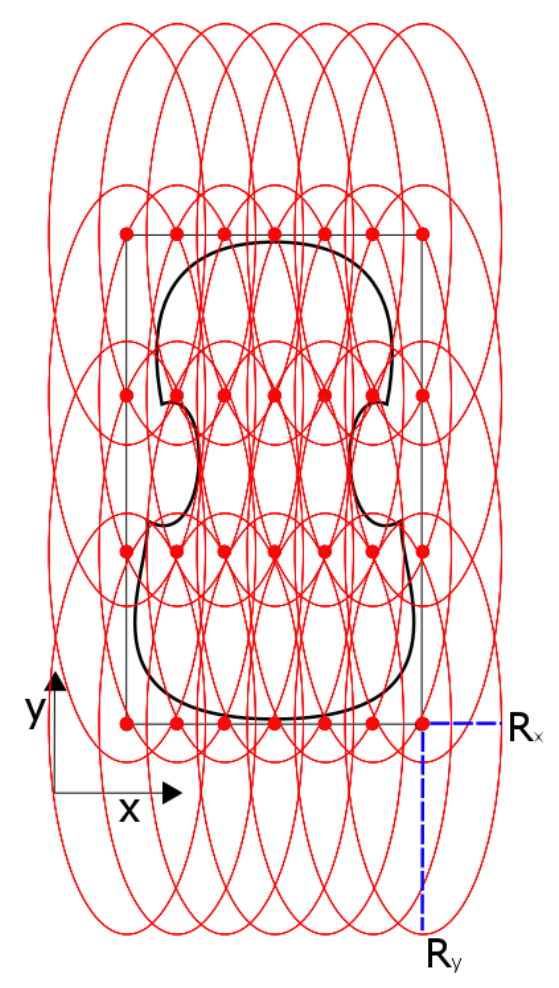

Figure 3: Orientation of the basis functions over the violin body.

these parameters independent intervals are assigned. The exponents $p_{1}$ and $p_{2}$ are intervals that model the uncertainty on the decrease rate of the MFA across the plate. The interval bounds for the spruce wood used in the top plate are given in table 6. The absolute bounds of MFA across the wood are $[2,14]$.

For the modelling of the uncertainty on the density, we use an interval representation introduced by the authors in [20], which includes the spatial dependency of the density by setting bounds on the maximal gradient of the density. The general definition is given in eq. 6 :

$$
\rho^{I}(x, y)=\rho_{0} \cdot\left(1+\sum_{i=1}^{n} \alpha_{i}^{I} \cdot \phi_{i}(x, y)\right)
$$

In this definition, $\phi_{i}(s, y)$ are radial basis functions. Each function is associated with a separate interval parameter, due to the overlap of the basis functions the interval parameters can be assumed independent while still obeying a constraint on the maximum gradient of the field. Their orientation is illustrated in figure 3 . Figure 4 shows such a basis function. 


\begin{tabular}{ccc}
\hline parameter in MFA model & lower bound & upper bound \\
\hline$\theta_{1}$ & 2 & 10 \\
$\theta_{2}$ & 2 & 10 \\
$\Delta \theta$ & 0 & 4 \\
$p_{1}$ & -0.25 & 0.25 \\
$p_{2}$ & -0.25 & 0.25 \\
\hline
\end{tabular}

Table 1: interval bounds in the MFA model

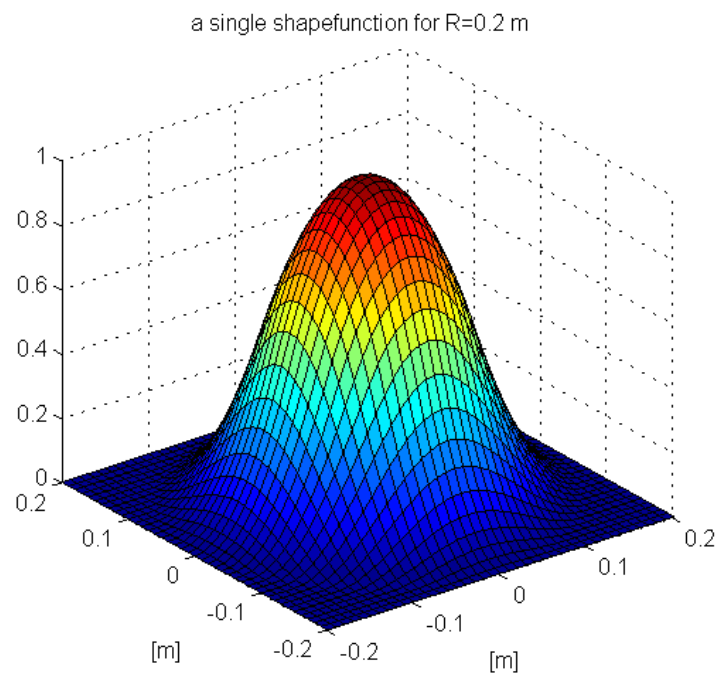

Figure 4: Example of a basis function.

For the z-value, a piecewise continuous second order polynomial function is used:

$$
z(r, \theta)=a \cdot\left\{\begin{array}{cc}
2\left(1-\frac{r}{R_{\max }(\theta)}\right)^{2} & 1>\frac{r}{R_{\max }(\theta)}>0.5 \\
1-2\left(\frac{r}{R_{\max }(\theta)}\right)^{2} & \frac{r}{R_{\max }(\theta)} \leq 0.5 \\
0 & \frac{r}{R_{\max }(\theta)}>1
\end{array}\right.
$$

, with $r=\sqrt{\left(x-x_{0}\right)^{2}+\left(y-y_{0}\right)^{2}}$ the distance from the central point and $R_{\max }(\theta)$ the maximum radius of the basis function in a certain direction. For the top plate, a definition of 56 basis functions is used. The base functions used have a different radius in $\mathrm{x}$ - and $\mathrm{y}$-direction, as can be seen from figure 3. The variation of the density is known to vary more in the radial direction as in the longitudinal direction, simply due to the fact that changing weather conditions manifest themselves across the tree's ageing rings in the radial direction. For this, we will define separate radii $R_{L}$ and $R_{r}$ for the longitudinal and radial directions, leading to oval shaped basis functions, the bases of which are illustrated in figure 3. By choosing the scaling factor $a$, the function radii $R_{L}$ and $R_{r}$ and the distances between the center points $\Delta x$ and $\Delta y$, we can set the maximum gradient $\left(\frac{\partial f}{\partial x}, \frac{\partial f}{\partial y}\right)$ as well as the maximum deviation $U$ in each point. The relations are given below in equation 8 . The $\mathrm{x}$-axis is set along the radial direction, the $\mathrm{y}$-axis is set along the longitudinal direction. 

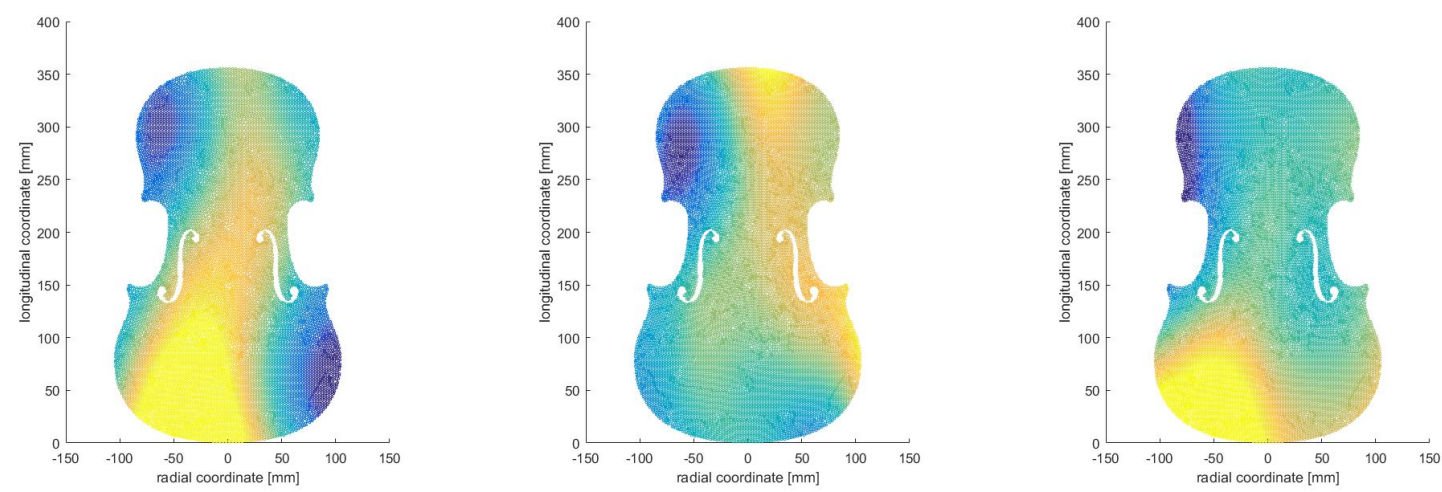

Figure 5: some examples of density distributions over the violin top plate
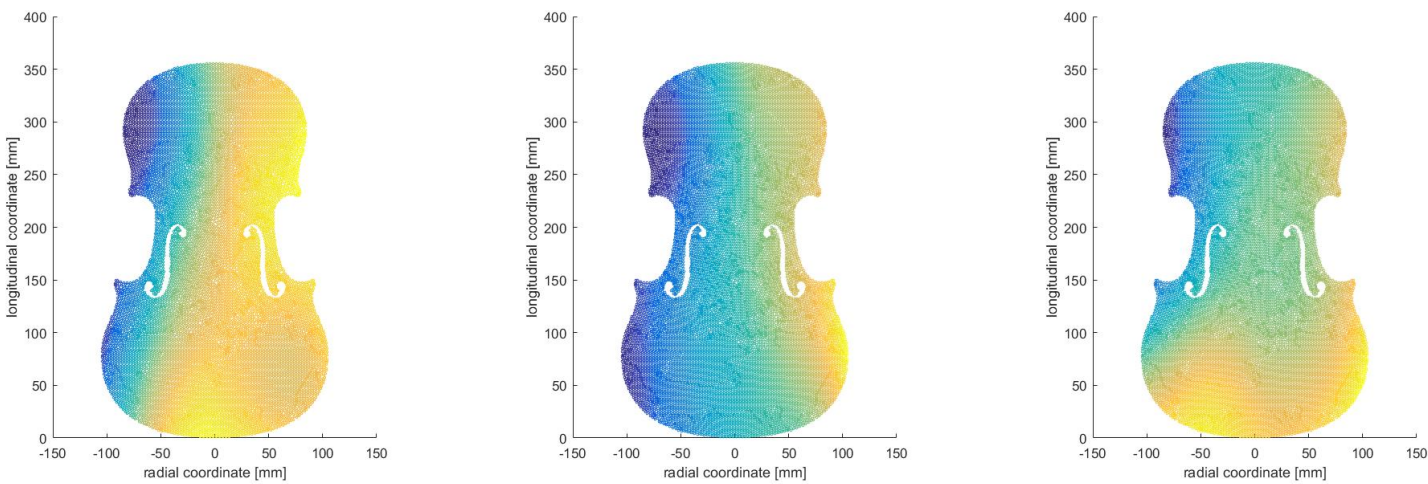

Figure 6: some examples of longitudinal stiffness distributions over the violin top plate

$$
\begin{array}{r}
U=\frac{7 \pi}{24} \cdot \frac{R_{r}}{\Delta x} \frac{R_{L}}{\Delta y} \\
\frac{\partial f}{\partial y}=\frac{2 a R_{r}}{\Delta x \Delta y} \frac{\partial f}{\partial x}=\frac{2 a R_{L}}{\Delta x \Delta y}
\end{array}
$$

the choice of $\Delta x$ and $\Delta y$ depend on the choice of $R_{L}$ and $R_{r}$, as equation 8 only holds for sufficiently large values of $\frac{R_{L}}{\Delta y}$ and $\frac{R_{r}}{\Delta x}$. A $20 \%$ deviation on $\rho$ corresponds to $U=0.2$, a maximum (relative) gradient of $(0.00182,0.00365) \mathrm{mm}^{-1}$ corresponds to $R_{L}=240 \mathrm{~mm}, R_{r}=$ $120 \mathrm{~mm}$ and $a=0.038 . \Delta x$ and $\Delta y$ are set at $50 \mathrm{~mm}$ and $100 \mathrm{~mm}$ respectively.

The bases of the basis functions are illustrated in figure 3. Note that the central point of some functions are located outside of the domain of the plate, but are still included as a term in equation 6 because a part of their nonzero domain is located on the plate. Figures 5,6 and 7 show some random realisations of the top plate by randomly sampling the intervals in equations 5 and 6, showing the dependency present in both the stiffness and the density.

\section{PROPAGATION}

In order to accurately propagate the effect of the density, we will use the spatial information of the density interval parameters. Allthough we assume the interval parameters can vary independently, their effects on the violin body natural frequencies are probably highly similar. To account for this, we make use of so called coefficient fields. We know from the basic me- 

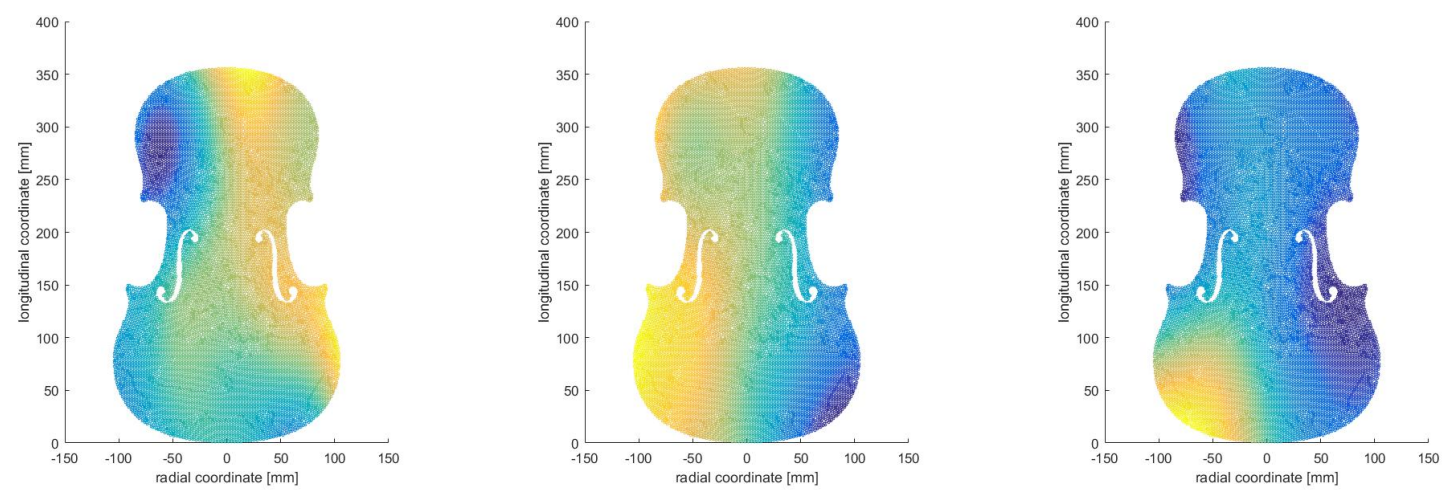

Figure 7: some examples of radial stiffness distributions over the violin top plate

chanics of plates that the square of the eigenfrequency is proportional to the specific stiffness $\frac{E}{\rho}$ (equation 9).

$$
\omega_{i}^{2}=a_{L} \cdot \frac{E_{L}}{\rho}+a_{R} \cdot \frac{E_{R}}{\rho}
$$

In the case of varying properties, we can again create a similar expression for the density in each field point:

$$
d\left(\omega_{i}^{2}\right)=a_{L}(x, y) \cdot \frac{E_{L}(x, y)}{\rho(x, y)}+a_{R}(x, y) \cdot \frac{E_{R}(x, y)}{\rho(x, y)}
$$

in this equation $d\left(\omega_{i}^{2}\right)$ represents the change in eigenfrequency due to a varying density in a single. Simply integrating this equation over the entire plate then leads to the following expression:

$$
\omega_{i}=\sqrt{\int_{x, y} d\left(\omega_{i}^{2}\right)}=\sqrt{\int_{x, y} a_{L}(x, y) \cdot \frac{E_{L}(x, y)}{\rho(x, y)} d x d y+\int_{x, y} a_{R}(x, y) \cdot \frac{E_{R}(x, y)}{\rho(x, y)} d x d y}
$$

In this expression, the coefficients of the polynomial model are given a spatial character. Previous research [21] indicated that for low order polynomials applied to plate based models, these coefficient fields exhibit a high degree of continuity, because of the correlated behaviour of the eigenfrequencies with respect to changing density in different points on the plate. This allows us to in turn model these coefficient fields by low order polynomials:

$$
a(x, y)=q_{1122} x^{2} y^{2}+q_{122} x y^{2}+q_{112} x^{2} y+q_{11} x^{2}+q_{22} y^{2}+q_{12} x y+q_{1} x+q_{2} y+q_{0}
$$

This allows us to drastically reduce the true dimension of the problem by immediately focussing on the coefficients of equation 12, rather than identifying a relationship for each field point separately. For this problem, the 15000 discrete coefficients that would have to be determined for the specific stiffness variation are then replaced by only 9 coefficients that define the continuous coefficient field. Once the model of equation 11 is known, the relationship of 
the parameters governing the MFA can then be inferred from equation 3 . This leads to two coefficient fields with 9 coefficients each, so a total of 18 parameters have to be determined.

A very important remark to make here is that as the order of the polynomial in eq. 9 increases, the applicability of this approach decreases, as the continuity of the coefficient fields rapidly decreases for higher order terms. More on this can be found in [21, 22].

\section{RESULTS}

For the training of the metamodel with the coefficient fields, 600 random experiments are used. After the creation of the metamodel, 50000 samples are generated to estimate the uncertainty on the frequencies. The coefficient fields are shown in figures 8 and 9 , for both the specific longitudinal stiffness and the specific radial stiffness. Both are in the same order of magnitude, indicating that both the longitudinal and radial stiffnesses are of important influence on the natural violin plate frequencies. The high degree of symmetry corresponds to our expectations as the associated mode shapes are symmetrical as well. On figures 10,11 and 12 , the uncertain domain is shown as the $2 \mathrm{D}$ projections of the $\left(f_{1}, f_{2}, f_{5}\right)$-domain. The samples of the FE-solver are marked in red (600 in total), the 50000 samples from the coefficient field model are marked in blue and serve as an estimate of the uncertain region. We see that $20 \%$ density variation combined with the uncertain MFA lead to a maximum frequency variation of about $10 \%$. We also see positive correlation of the first and second eigenfrequency, which from the mode shapes seems feasible. But also the coefficient fields of figure 8 support this, as the spatial effect of the longitudinal stiffness on both the first and second eigenfrequency is similar. However, striking is the negative correlating between mode shape 2 and 5, which we believe is due to the way the longitudinal and radial stiffnesses are related. Because of the model used in equation 3, both have opposite relations with respect to the MFA, and the frequency of the 5th mode appears to decrease drastically with increasing MFA, much more than mode 2.
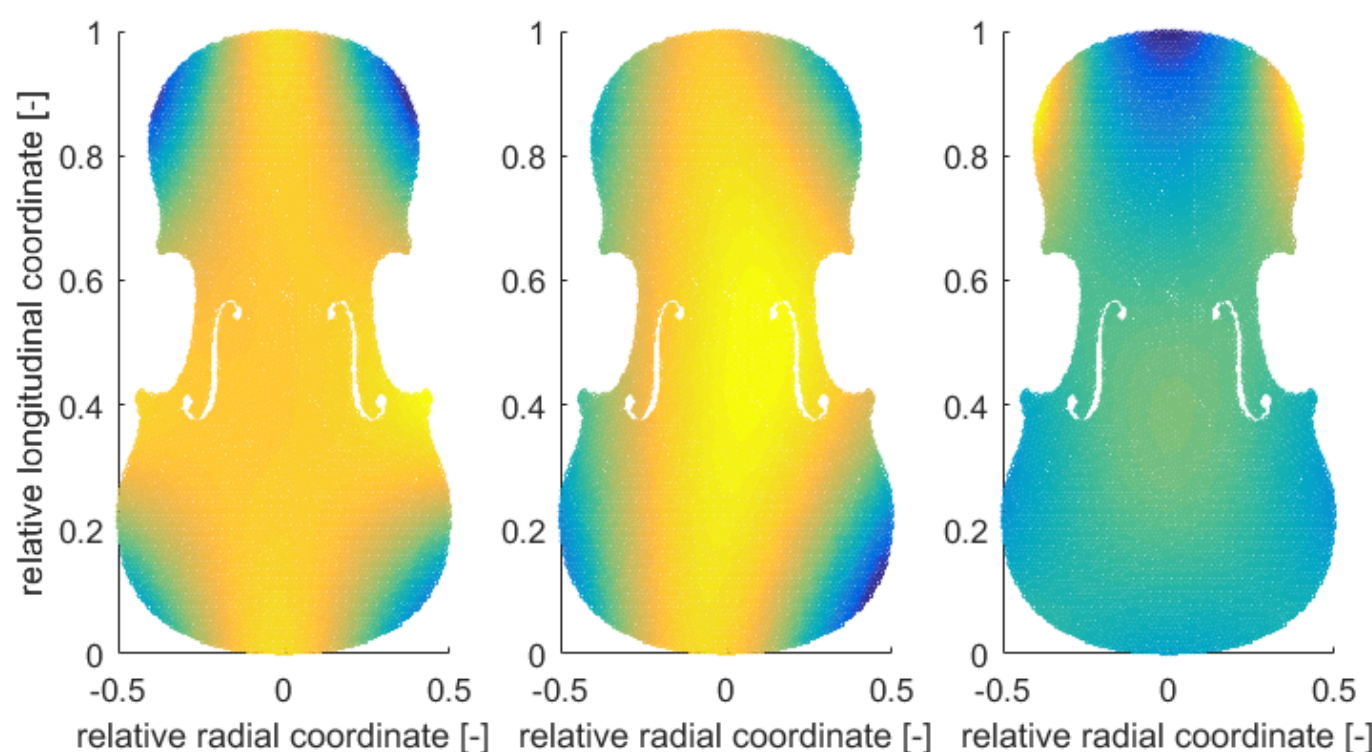

relative radial coordinate $[-]$ relative radial coordinate $[-]$

Figure 8: coefficient fields for the three output frequencies for the longitudinal stiffness 

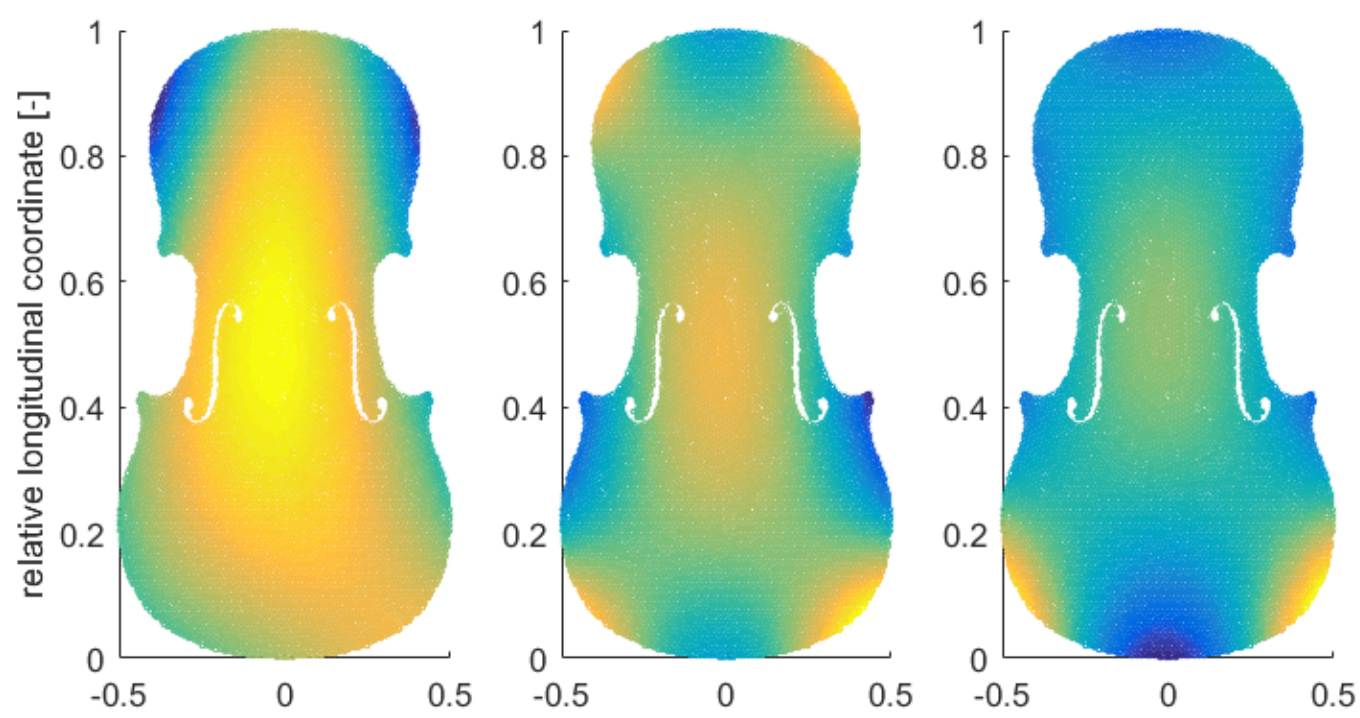

relative radial coordinate $[-]$ relative radial coordinate $[-]$ relative radial coordinate $[-]$

Figure 9: coefficient fields for the three output frequencies for the radial stiffness

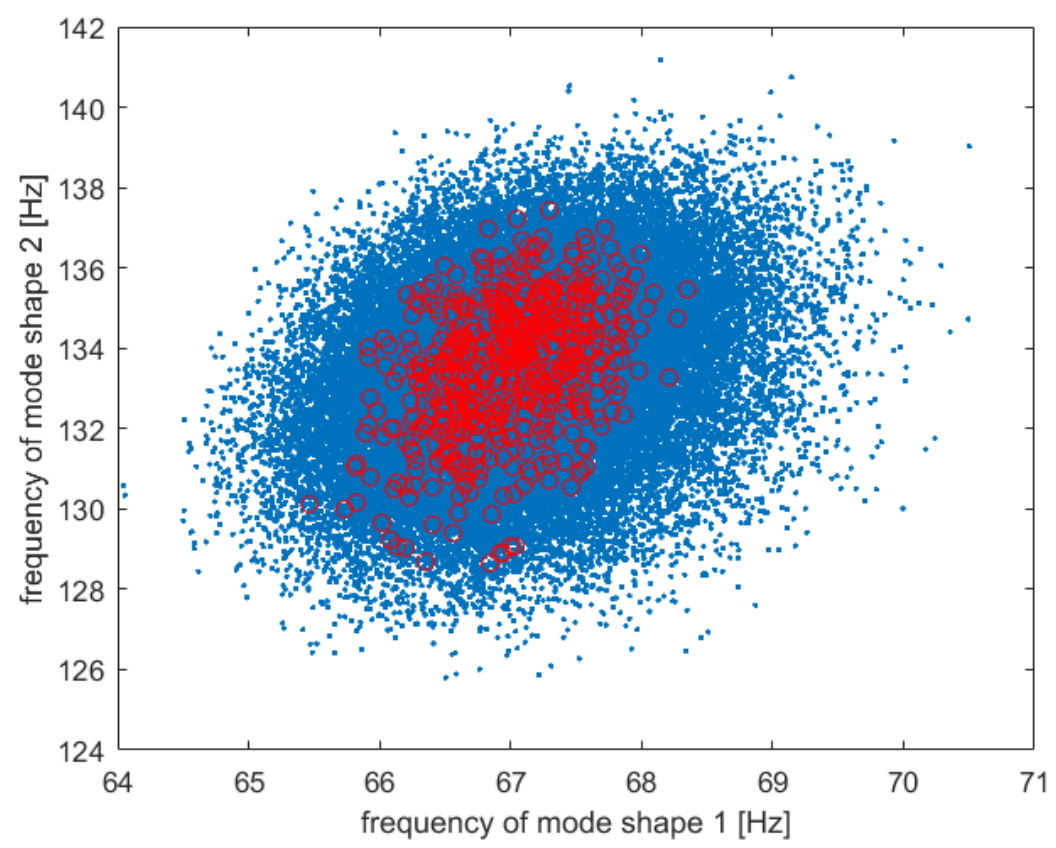

Figure 10: uncertainty region projected on the $\left(f_{1}, f_{2}\right)$-plane 


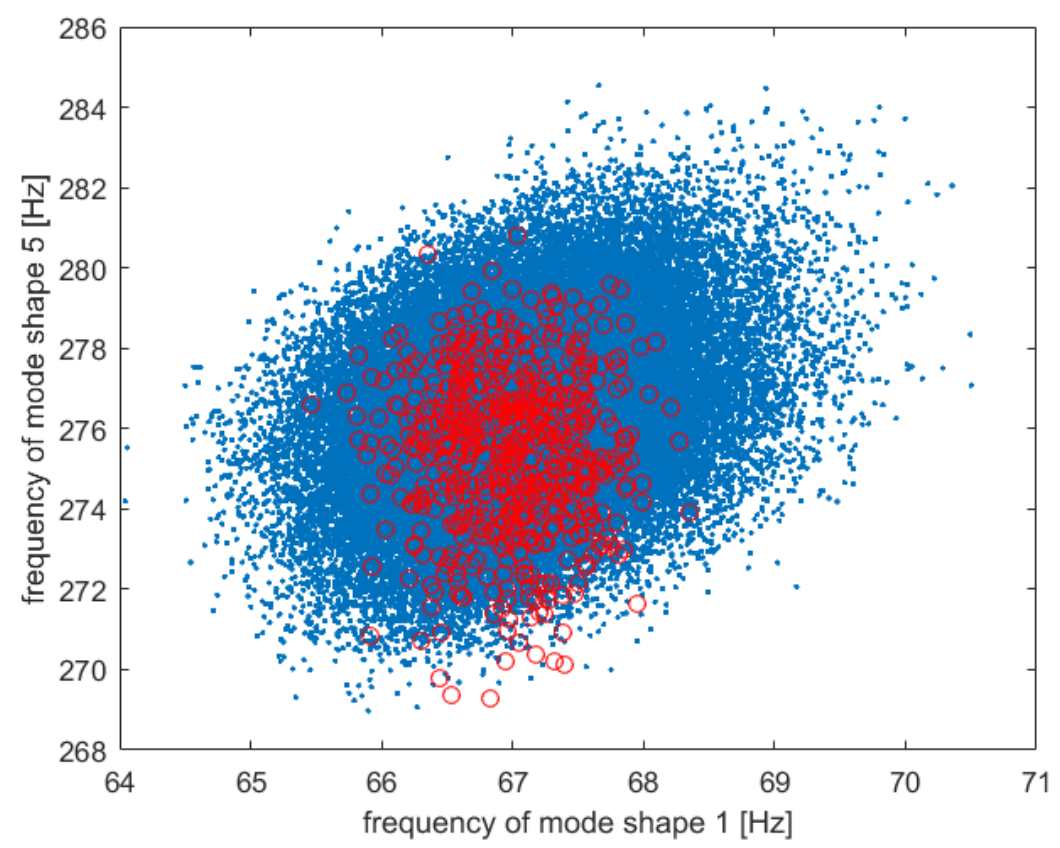

Figure 11: uncertainty region projected on the $\left(f_{1}, f_{5}\right)$-plane

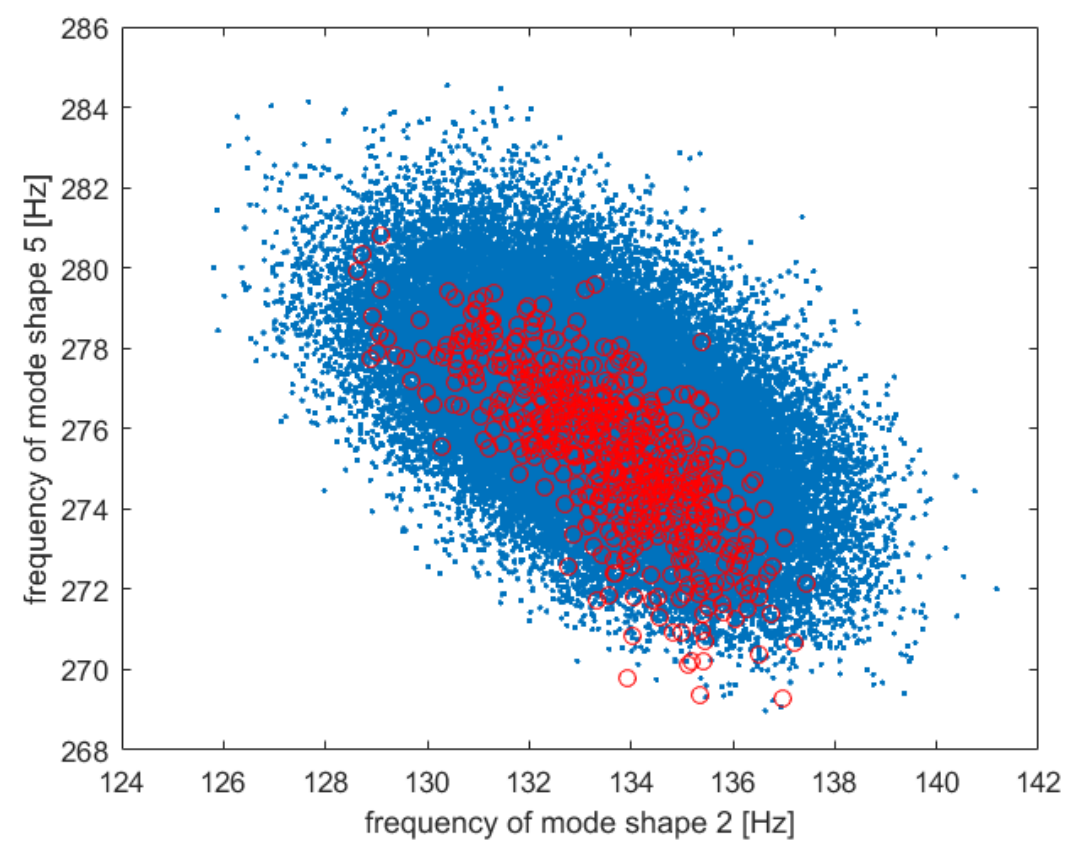

Figure 12: uncertainty region projected on the $\left(f_{2}, f_{5}\right)$-plane

\section{CONCLUSIONS}

In this paper, the interval field method has been applied on a large model of a violin top plate to assess the natural frequency variation due to uncertain structural properties of the wood. The 
uncertainty was estimated based on the wood selection process in violin construction. Literature data was used to feed an interval field model that accounts for dependency of the density parameter, which was different in radial and longitudinal direction. The stiffnesses were related to the MFA through a simple linear relationship.

Future steps will be taken to feed this model with more accurate estimations of the density and the MFA through acquiring experimental data with the emphasis on spatial variability. Also, the relation of MFA and stiffness shall be further investigated as the linear relationship is likely to be too simple. However, the theory of interval fields provides an effective tool to include whatever information there may be on dependency and spatial uncertainty.

\section{ACKNOWLEDGEMENTS}

The research fund of the KU Leuven als well as the fund for scientific research (F.W.O.) are gracefully acknowledged for their support.

\section{REFERENCES}

[1] E. V. Jansson, Experiments with the Violin string and bridge, Applied Acoustics, Vol. 30. $133-146,1990$

[2] E. V. Jansson, Violin frequency response - bridge mobility and bridge feet distance, Applied Acoustics, Vol. 65. 1197 - 1205, 2004

[3] T. D. Rossing, The Sience of String Instruments, Springer Science+Business Media, 2010

[4] , G. Bissinger, The role of radiation dampinh in violin sound, Acoustics Research Letters Online Vol. 5(3), 82 - 87, 2004

[5] , G. Bissinger, Radiation damping, efficiency, and directivity for violin normal modes below 4 kHz, Acoustics Research Letters Online Vol. 4(1), 7 - 12, 2003

[6] G. Bissinger, Modal analysis of a violin octet, The Journal of the Acoustical Society of America, Vol. 113(4), 2015 - 2113, 2003

[7] K. D. Marshall, Modal analysis of a violin, The Journal of the Acoustical Society of America, Vol. 77(2), $695-709,1985$

[8] E. V. Jansson, acoustic for violin and guitar makers, 4th edition, KTH, Dept. of Speech, Music an Hearing, 2002

[9] V. Bucur, Acoustics of Wood, 2nd edition, Springer-Verlag, 2006

[10] I. Bremaud, What do we know on "resonance wood" properties? Selective review and ongoing research. Porceedings of the Acoustics 2012 Conference, 2759 - 2764, Nantes, France

[11] W. Molinski, E. Roszyk, J. Puszynski, Variation in Mechanical Properties within individual annual rings of the resonance spruce wood, DRVNA INDUSTRIJA VOL. 65(3), 215 233,2014 
[12] M. Spycher, F. W. Schwarze, R. Steiger, Assessment of resoance wood quality by comparing its physical and histological properties, Wood Science Technology, Vol. 42, 325 - 342, 2008

[13] C. Buksnowitz, A. Teischinger, U. Mller, A. Pahler, R. Evans, Resonance wood [Picea abies (L.) Karst.] - evaluation and prediction of ivolin makers' quality-grading, The Journal of the Acoustical Society of America, Vol. 121(4), 2384 - 2395, 2007

[14] C. Carlier, I. Bremaud, J. Grill, Violin making "tonewood": comparing makers' empirical expertise with wood structural/visual and acoustical properties, Proceedings of ISMA2014 325 -330, Le Mans, France

[15] R. Hori, M. Mller, U. Watanabe, H. C. Lichtenegger, P. Fratzl, J. Sugiyama, The importance of seasonal differences in the cellulose microfibril angle in softwoods in determining acousic properties, Journal of Material Science, Vol. 37, 4279 - 4284, 2002

[16] T. Jyske, H. Mkinen, P. Saranp, Wood density within Norway Spruce Stems, Silva Fennica, Vol. 42(3), 439 - 455, 2008

[17] , V. Gryc, P. Horacek, Variability in density of spruce (Picea abies [L.] Karst.) wood with the presence of reaction wood, Journal of Forest Science, Vol. 53(3), 129 - 137, 2007

[18] I. Sopushynskyy, I. Kharyton, A. Teischinger, V. Mayevskyy, H. Hrynyk, Wood density and annual growth variability of Picea abies (L.) Karst. growing in the Ukrainian Carpathians, European Journal of Wood and Wood Products, 1 - 10, 2016

[19] M. Treacy, J. Evertsen, A. Ni Dhubhain, A comparison of mechanial and physical wood properties, 1st. edition, COFORD, 2000

[20] M. Imholz, D. Vandepitte, D. Moens, Derivation of an input interval field decomposition based on expert knowledge using locally defined basis functions, proceedings of UNCECOMP2015 international conference on Uncertainty Quantification in Computational Sciences and Engineering, Hersonissos, Greece

[21] M. Imholz, D. Vandepitte, D. Moens, Application of interval fields to geometric output quantities of finite element models, proceedings of UNCERTAINTIES2016 International Symposium on Uncertainty Quantification and Stochastic Modelling, Maresias, Brazil

[22] M. Imholz, D. Vandepitte, D. Moens, Dynamical analysis of plate models with uncertainty structural properties using the interval field method, proceedings of ECCOMAS2016 European congress on Computational Methods in Applied Sciences and Engineering, Hersonissos, Greece 